\title{
Design of Augmented Nonlinear PD Controller of Delta/Par4-Like Robot
}

\author{
Amjad J. Humaidi (i) and Ahmed Ibraheem Abdulkareem \\ Control and Systems Engineering Department, University of Technology, Baghdad, Iraq \\ Correspondence should be addressed to Amjad J. Humaidi; 601116@uotechnology.edu.iq
}

Received 7 March 2019; Accepted 9 May 2019; Published 1 July 2019

Guest Editor: Wei Zhang

Copyright (C) 2019 Amjad J. Humaidi and Ahmed Ibraheem Abdulkareem. This is an open access article distributed under the Creative Commons Attribution License, which permits unrestricted use, distribution, and reproduction in any medium, provided the original work is properly cited.

\begin{abstract}
This work presents the design of two control schemes for a Delta/Par4-like parallel robot: augmented PD (APD) controller and augmented nonlinear PD (ANPD) controller. The stability of parallel robot based on nonlinear PD controller has been analyzed and proved based on Lyapunov method. A comparison study between APD and ANPD controllers has been made in terms of performance and accuracy improvement of trajectory tracking. Also, another comparison study has been presented between augmented nonlinear PD (ANPD) controller and nonaugmented nonlinear PD (NANPD) controller in order to show the enhancement of introducing the augmented structure on dynamic performance and trajectory tracking accuracy. The effectiveness of augmented PD controllers (APD and ANPD) and nonaugmented nonlinear PD (NANPD) controller for the considered parallel robot are verified via simulation within the MATLAB environment.
\end{abstract}

\section{Introduction}

The parallel manipulators are defined as mechanisms with closed-loop kinematic chains, in which the end effector is linked to the base through several independent kinematic chains. Parallel manipulator has the advantages of high speed, high precision, and ability to manipulate heavy loads [1].

In the last two decades, several structures of modified proportional integral derivative (PID) controllers have been presented in the industrial control application. One of these controllers is the nonlinear PID (NPID), which is introduced by HAN [2]. The main idea was to replace the gain scheduling by a nonlinear gain function by introducing a continuous dynamic nonlinear function to achieve better noise rejection and better tracking. This is achieved by synthesizing a function composed of a linear function near the zero error and nonlinear function far from zero error.

Recently, several controllers are proposed to control the parallel manipulator. In [3], Anoop R Nair and Achu Govind KR designed and verified the performance of adaptive PID control of Delta parallel manipulator. In this work, the controller was able to trace the desired trajectory without any deflection. Zhang et al. studied the problem of dynamic control design for redundantly actuated planer 2-DOF parallel manipulator. The work proposed an augmented PD controller based on forward dynamic compensation control technique, which showed better performance when compared with conventional PD controller [4]. Hussein Saied et al. proposed different model-based (augmented PD and adaptive feedforward with PD) controllers and nonmodel-based (PD, PID, and nonlinear PD) controllers for a 4-DOF parallel VELOCE robot [5]. The work investigated how well the dynamic performance of the system could be enhanced by including Parallel Kinematic Manipulator (PKM) and employing its varied parameters for further improvement. Wei-Wei Shang et al. proposed an augmented nonlinear PD controller based on conventional dynamic control. This proposed controller replaced linear PD controller in order to improve the tracking accuracy for 2 DOF redundantly actuated parallel manipulator [6]. Wei-Wei Shang and Shuang Cong developed new computed torque (CT) controllers called nonlinear computed torque (NCT), which is applied to real high-speed planer parallel [7]. Su et al. developed a nonlinear proportional integral derivative (N-PID) algorithm in link space to achieve high precision tracking control for a general 6-DOF parallel manipulator. Experimental results indicated that the nonlinear control method can achieve a superior performance [8]. Fatma et al. designed nonlinear PID controller for trajectory 


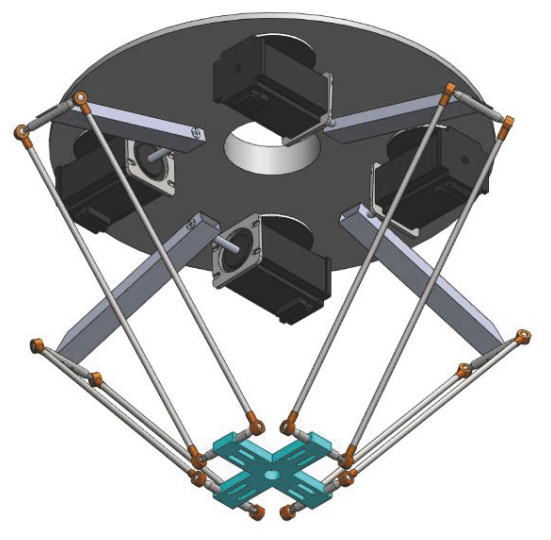

FIGURE 1: Solid work drawing of Delta/Par4-like parallel manipulator.

tracking of a manipulator robot (SCARA) and developed a PID controller having high performances in terms of controllability and stability of manipulator [9].

In the present work, design and stability analysis of augmented-based PD control structure is presented in order to control a redundant Delta/Par4-like parallel manipulator.

Delta/Par4-like robot is a redundantly actuated parallel manipulator and it is much known for the application that needs for high speed and high acceleration. The Delta/Par4like robot is characterized by light-weight mechanical component common with good structural stiffness. Also, it can achieve velocity and acceleration to reach above $10 \mathrm{~m} / \mathrm{s}$ and $150 \mathrm{~m} / \mathrm{s}^{2}$, respectively, in case of pick and place applications. This redundant actuated parallel manipulator is able to reach $100 \mathrm{G}$. This parallel robot is 3-DOF (translation along $\mathrm{x}-\mathrm{y}$ z) and has four actuators (redundantly actuated), each motor has a maximum torque of $127 \mathrm{~N}$.m. The suggested parallel manipulator has a workspace of at least a cylinder of $300 \mathrm{~mm}$ radius and $100 \mathrm{~mm}$ height [10]. Figure 1 shows an outlook of Delta/Par4-like robot.

The main contribution of the work can be summarized by the following points:

(i) Design of augmented-based PD control scheme for controlling a redundant Delta/Par4-like parallel robot.

(ii) Performance comparison between augmented-based controllers (APD and ANPD) in terms of dynamic behaviors and accuracy of trajectory tracking.

(iii) A Lyapunov-based stability analysis is presented for parallel robot controlled by ANPD controller to prove the asymptotically convergence of both tracking error and error rate to zero as $t \longrightarrow \infty$.

(iv) A comparison study has been made between augmented-based nonlinear PD (ANPD) controller and nonaugmented-based nonlinear PD (NANPD) controller in terms of tracking accuracy and dynamic performance.

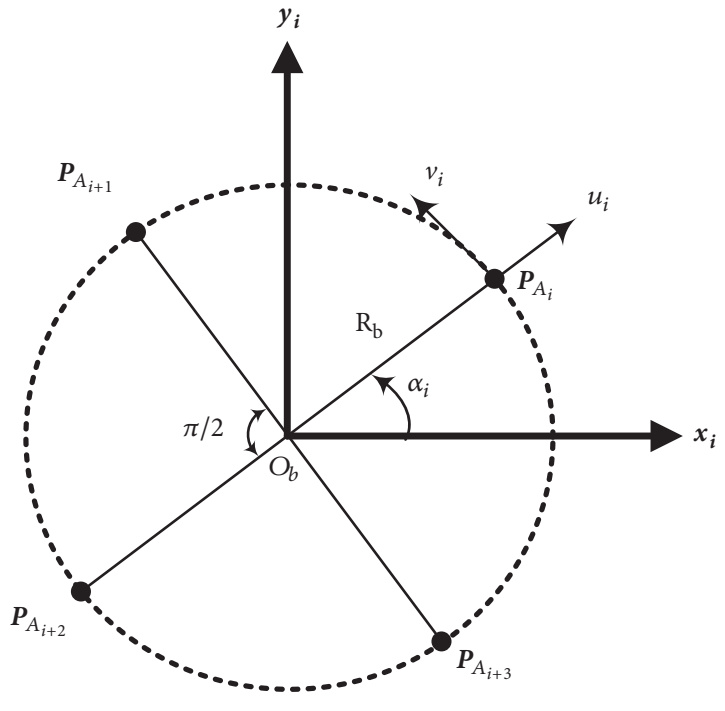

(a) Top view

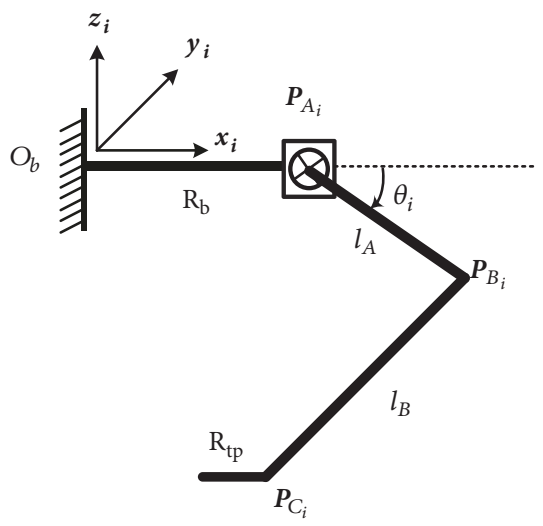

(b) Side view

Figure 2: Geometric parameters of Delta/Par4-like robot.

\section{Geometric Description of Delta/Par4-Like Robot}

The geometric parameters used in describing the dynamic model of Delta/Par4-like robots are depicted in Figure 2, where $i=1, . ., 4$. In developing the dynamic model of parallel robot, the forearms of Delta/Par4-like parallel robot are replaced by simple rods.

The Cartesian coordinates $x-y-z$ of joint center locations $P_{A_{i}}, P_{C_{i}}$, and $P_{B_{i}}$ are

$$
\begin{aligned}
\boldsymbol{P}_{A_{i}} & =\left[\begin{array}{lll}
x_{-} P_{A_{i}} & y \_P_{A_{i}} & z P_{A_{i}}
\end{array}\right]^{T}=R_{b}\left[\begin{array}{lll}
\cos \alpha_{i} & \sin \alpha_{i} & 0
\end{array}\right]^{T} \\
\boldsymbol{P}_{B_{i}} & =\left[\begin{array}{lll}
x_{-} P_{B_{i}} & y_{-} P_{B_{i}} & z_{-} P_{B_{i}}
\end{array}\right]^{T} \\
& =\boldsymbol{P}_{A_{i}}+l_{A}\left[\begin{array}{lll}
\cos \alpha_{i} \cos q_{i} & \sin \alpha_{i} \cos q_{i} \sin q_{i}
\end{array}\right]^{T} \\
\boldsymbol{P}_{C_{i}} & =\left[\begin{array}{lll}
x_{-} P_{C_{i}} & y_{-} P_{C_{i}} & z_{-} P_{C_{i}}
\end{array}\right]^{T} \\
& =\left[\begin{array}{lll}
R_{t p} \cos \alpha_{i}+x & R_{t p} \sin \alpha_{i}+y & z
\end{array}\right]^{T}
\end{aligned}
$$


where $R_{t p}$ and $R_{b}$ are the radius of traveling plate and base of robot, respectively, $l_{A}$ and $l_{B}$ are the lengths of arm and forearm, respectively, and $\alpha_{i}$ denotes the orientation of actuator axes, described by

$$
\alpha_{i}=\frac{2(i-1) \pi}{4}, \quad i=1, \ldots 4
$$

There is another frame associated with each actuator designated as $P_{i}-\boldsymbol{u}_{i} \boldsymbol{v}_{i} \boldsymbol{z}$, where $\boldsymbol{u}_{i}$ and $\boldsymbol{v}_{i}$ are given by

$$
\begin{aligned}
& \boldsymbol{u}_{i}=\left[\begin{array}{lll}
\cos \alpha_{i} & \sin \alpha_{i} & 0
\end{array}\right]^{T} \\
& \boldsymbol{v}_{i}=\left[\begin{array}{lll}
-\sin \alpha_{i} & \cos \alpha_{i} & 0
\end{array}\right]^{T}
\end{aligned}
$$

The relation between Cartesian and joint velocity vectors of the traveling plate is described in the following equation [11]:

$$
J_{q} \dot{\mathbf{q}}=J_{x} \dot{X}
$$

where $\dot{\mathbf{q}}$ and $\dot{\boldsymbol{X}}$ are the joint and Cartesian velocity vectors, respectively. Equation (4) can be written as follows:

$$
\dot{\mathbf{q}}=\boldsymbol{J}_{q}^{-1} \boldsymbol{J}_{x} \dot{\boldsymbol{X}}=\boldsymbol{J}_{m} \dot{\boldsymbol{X}}
$$

Therefore, the matrix $\boldsymbol{J}_{m}$ maps the velocity vector of the forearm from joint to Cartesian space. The matrix $J_{m}$ is square and invertible in case of nonredundant robots, while it is not inverted for redundant robots and the pseudoinverse matrix can be used instead. The derivation of (5) leads to

$$
\ddot{\boldsymbol{q}}=\boldsymbol{J}_{m} \ddot{\boldsymbol{X}}+\dot{\boldsymbol{J}}_{m} \dot{\boldsymbol{X}}
$$

\section{The Simplified Dynamic Model of Delta/Par4-Like Robot}

This section presents the simplified direct dynamic model (DDM) of Delta/Par4-like parallel manipulators. To do so, the following assumptions are made $[12,13]$ :

(1) Neglect the joint friction.

(2) Neglect the inertia of forearms $\left(I_{f} \cong 0\right)$.

(3) The total mass of each forearm $m_{f}$ is assumed to be split into two parts located at the ends of the forearm such that each part has half the value of $m_{f}$ as indicated in Figure 3.

(4) Due to very high acceleration, which reaches up to $100 \mathrm{G}$, the gravity will not be considered and can be neglected.

To start the derivation of simplified dynamic model of Delta/Par4-like robot, the equilibrium analysis of the arm is first presented. The actuator torque vector $\boldsymbol{\tau}_{q}$ is related to the acceleration vector $\ddot{\boldsymbol{q}}$ by the following equation:

$$
\boldsymbol{\tau}_{q}-\boldsymbol{J}_{q}^{T} \boldsymbol{F}=\boldsymbol{I}_{t o t} \ddot{\boldsymbol{q}}
$$

where $\boldsymbol{J}_{q}^{T}$ denotes the joint Jacobian of the robot, $\boldsymbol{\tau}_{q}$ is the vector of actual joint torques, $\boldsymbol{F}$ represents the vector of forces

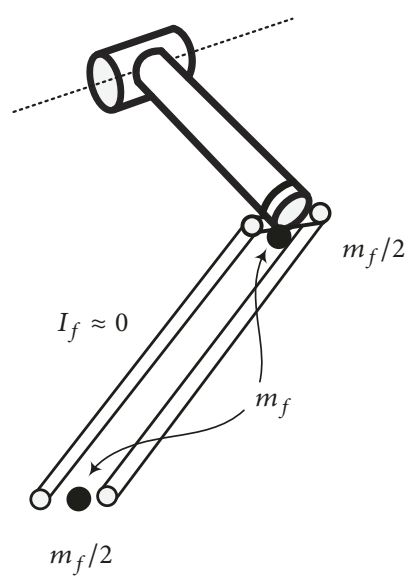

FIGURE 3: Splitting the forearm mass into two parts.

exerted on robot arm at points $P_{A_{i}}$, and $\boldsymbol{I}_{\text {tot }}$ is a diagonal matrix whose diagonal elements is given by

$$
\boldsymbol{I}_{\text {tot }}=I_{a c t}+I_{\text {arm }}+\frac{\left(m_{f} l_{A}^{2}\right)}{2}
$$

where $\boldsymbol{I}_{\text {arm }}$ and $\boldsymbol{I}_{a c t}$ are the inertia of arms and actuator, $l_{A}$ defines the length of the arm, and $\boldsymbol{m}_{f}$ is the mass of the forearm.

Secondly, the equilibrium of the traveling plate is analyzed. The motion equation of the traveling plate is given by [10]

$$
\boldsymbol{J}_{x}^{T} \boldsymbol{F}=\boldsymbol{M}_{t o t} \ddot{\boldsymbol{X}}
$$

where $\boldsymbol{J}_{x}^{T}$ is Cartesian Jacobian of the robot and $\boldsymbol{M}_{\text {tot }}$ is a diagonal matrix whose elements are given by

$$
\boldsymbol{M}_{t o t}=m_{t p}+\mathrm{n} \frac{m_{f}}{2}
$$

where $n$ denotes the number of actuators and $m_{t p}$ is the traveling plate mass. The Cartesian Jacobian $\boldsymbol{J}_{x}$ of Delta/Par 4 like parallel robots is not invertible if there are more actuators than degrees-of-freedom.

Substituting (7) into (9), the dynamic model of Delta/Par4 like robot will be written in Cartesian space as

$$
\boldsymbol{M}_{t o t} \ddot{\boldsymbol{X}}=\boldsymbol{J}_{x}^{T} \boldsymbol{J}_{q}^{-T}\left(\boldsymbol{\tau}_{q}-\boldsymbol{I}_{t o t} \ddot{\boldsymbol{q}}\right)=\boldsymbol{J}_{m}^{T}\left(\boldsymbol{\tau}_{q}-\boldsymbol{I}_{t o t} \ddot{\boldsymbol{q}}\right)
$$

Using (6), one can obtain

$$
\boldsymbol{M}_{t o t} \ddot{\boldsymbol{X}}=\boldsymbol{J}_{m}^{T}\left(\boldsymbol{\tau}_{q}-\boldsymbol{I}_{t o t}\left(\boldsymbol{J}_{m} \ddot{\boldsymbol{X}}+\dot{\boldsymbol{J}}_{m} \dot{\boldsymbol{X}}\right)\right)
$$

or

$$
\left(\boldsymbol{M}_{t o t}+\boldsymbol{J}_{m}^{T} \boldsymbol{I}_{t o t} \boldsymbol{J}_{m}\right) \ddot{\boldsymbol{X}}+\boldsymbol{J}_{m}^{T} \boldsymbol{I}_{t o t} \dot{\boldsymbol{J}}_{m} \dot{\boldsymbol{X}}=\boldsymbol{J}_{m}^{T} \boldsymbol{\tau}_{q}
$$

If one set $\boldsymbol{X}=\left[\begin{array}{lll}x & y & z\end{array}\right]$, then the dynamic model of (13) can be written in the following form:

$$
\boldsymbol{M} \ddot{\mathbf{X}}+\boldsymbol{C} \dot{\boldsymbol{X}}=\boldsymbol{J}_{m}^{T} \tau_{q}
$$




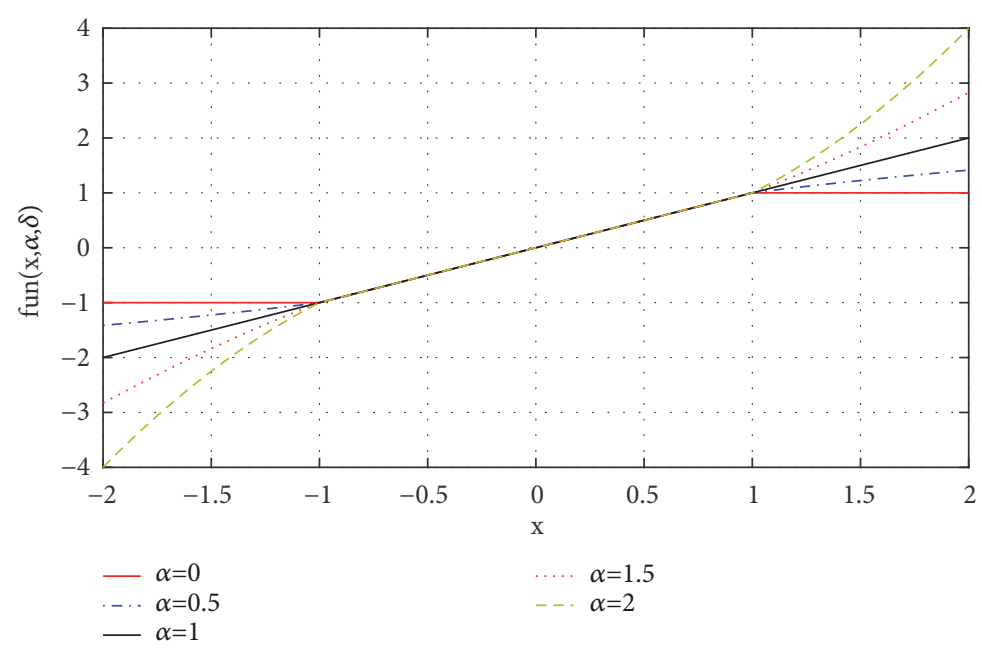

FIGURE 4: Graphical description of the nonlinear function.

or

$$
M \ddot{X}+C \dot{X}=F
$$

where $\boldsymbol{J}$ is the Jacobian matrix, $\mathbf{M}=\boldsymbol{M}_{t o t}+\boldsymbol{J}_{m}^{T} \boldsymbol{I}_{t o t} \boldsymbol{J}_{m}$ defines the inertial matrix, while $\boldsymbol{C}=\boldsymbol{J}_{m}^{T} \boldsymbol{I}_{\text {tot }} \dot{\boldsymbol{J}}_{m}$ stands for both centrifugal and Coriolis forces. The force matrix $\boldsymbol{F}$ expresses the forces required in the Cartesian task space and the following equation relates this force $\boldsymbol{F}$ to actual joint torque $\boldsymbol{\tau}_{q}$ :

$$
\boldsymbol{F}=\boldsymbol{J}_{m}^{T} \boldsymbol{\tau}_{q}
$$

Since the Delta/Par4-like manipulator is a redundant robot, the matrix $\boldsymbol{J}_{m}^{T}$ is not a square matrix and cannot be inverted. Hence, the pseudomatrix concept is involved and the pseudoinverse of $\boldsymbol{J}_{m}^{T}$ can be defined as

$$
\boldsymbol{H}^{T}=\boldsymbol{J}_{m}^{+T}=\left(\boldsymbol{J}_{m} \boldsymbol{J}_{m}^{T}\right)^{-1} \boldsymbol{J}_{m}
$$

Therefore, one can have the following expression, which relates the torque $\boldsymbol{\tau}_{q}$ to the force matrix $\boldsymbol{F}$ :

$$
\boldsymbol{\tau}_{q}=\boldsymbol{H}^{T} \boldsymbol{F}
$$

\section{Augmented NPD Control Design for Delta/Par4-Like Robot}

The ANPD controller proposed in the present work is synthesized by replacing the linear PD by the structure of APD controller based on NPD algorithm. The structure of the NPD controller can be described by [14]

$$
u_{T}(t)=k_{P} f u n\left(e(t), \alpha_{1}, \delta_{1}\right)+k_{d} f u n\left(\dot{e}(t), \alpha_{2}, \delta_{2}\right)
$$

The $f u n(e(t), \alpha, \delta)$ is a nonlinear function

$$
\text { fun }(e(t), \alpha, \delta)= \begin{cases}|e|^{\alpha} \operatorname{sign}(x) & |x|>\delta \\ \frac{x}{\delta^{1-\alpha}} & |x| \leq \delta\end{cases}
$$

where $\delta$ (Delta) determines the threshold of error (or error derivative), which discriminates between the linear (below or equal $\delta$ ) and nonlinear region (above $\delta$ ). The parameter $\alpha$ (alpha) governs the degree of nonlinearity and complexity of the function $f u n($.$) above the value of \delta$. The value of $\alpha$ is normally chosen between (0-2) and the function fun(.) has linear characteristics with $\alpha=1$ as illustrated in Figure 4.

According to general NPD control law of (19) and the dynamic model described by (15), the proposed NPD control law for Delta/Par4-like robot,

$$
\tau_{d}=\boldsymbol{M} \ddot{\boldsymbol{X}}^{d}+C \dot{\boldsymbol{X}}^{d}+\boldsymbol{K}_{p}(\boldsymbol{e}) \boldsymbol{e}+\boldsymbol{K}_{d}(\dot{\boldsymbol{e}}) \dot{\boldsymbol{e}}
$$

where $\dot{\boldsymbol{X}}^{d}$ and $\ddot{\boldsymbol{X}}^{d}$ are the desired velocity and desired acceleration of traveling plate, respectively, and $\boldsymbol{e}=\left[\begin{array}{lll}e_{x} & e_{y} & e_{z}\end{array}\right]^{T}=$ $\boldsymbol{X}^{d}-\boldsymbol{X}$ is the position error, $\boldsymbol{X}=\left[\begin{array}{lll}x & y & z\end{array}\right]^{T}$.

The control law of the ANPD controller of Delta/Par4like robot consists of two parts: the first part describes the dynamic compensation defined by the desired trajectory

$$
\tau_{d 1}=M \ddot{X}^{d}+C \dot{X}^{d}
$$

The second part represents the error elimination given by

$$
\boldsymbol{\tau}_{d 2}=\boldsymbol{K}_{p}(e) \boldsymbol{e}+\boldsymbol{K}_{d}(\dot{e}) \dot{\boldsymbol{e}}
$$

The nonlinear gains $\boldsymbol{K}_{p}(e)$ and $\boldsymbol{K}_{d}(\dot{e})$ are given by

$$
\begin{aligned}
\boldsymbol{K}_{p}(\boldsymbol{e}) & =\left[\begin{array}{ccc}
K_{p}\left(e_{x}\right) & 0 & 0 \\
0 & K_{p}\left(e_{y}\right) & 0 \\
0 & 0 & K_{p}\left(e_{z}\right)
\end{array}\right] \\
\boldsymbol{K}_{d}(\dot{\boldsymbol{e}}) & =\left[\begin{array}{ccc}
K_{d}\left(\dot{e}_{x}\right) & 0 & 0 \\
0 & K_{d}\left(\dot{e}_{y}\right) & 0 \\
0 & 0 & K_{d}\left(\dot{e}_{z}\right)
\end{array}\right]
\end{aligned}
$$




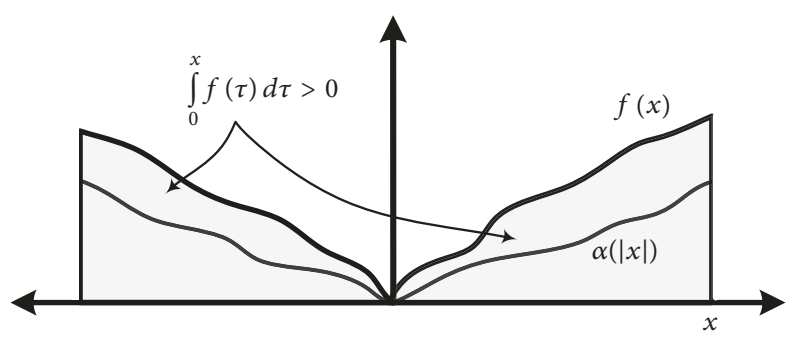

FIgURE 5: Graphical interpretation of Lemma 2.

where $K_{p}\left(e_{i}\right)$ and $K_{d}\left(\dot{e}_{i}\right)$ for $i=x, y, z$ are given by

$$
\begin{aligned}
& K_{p}\left(e_{i}\right)= \begin{cases}k_{p}\left|e_{i}\right|^{\alpha_{1}-1}, & \left|e_{i}\right|>\delta_{1} \\
k_{p} \delta_{1}^{\alpha_{1}-1}, & \left|e_{i}\right| \leq \delta_{1}\end{cases} \\
& K_{d}\left(\dot{e}_{i}\right)= \begin{cases}k_{d}\left|\dot{e}_{i}\right|^{\alpha_{2}-1}, & \left|\dot{e}_{i}\right|>\delta_{2} \\
k_{d} \delta_{2}^{\alpha_{2}-1}, & \left|\dot{e}_{i}\right| \leq \delta_{2},\end{cases}
\end{aligned}
$$

where $k_{p}$ and $k_{d}$ are positive constant gains.

\section{Stability Analysis of Delta/Par4-Like Robot Controlled by ANPD Controller}

This section focuses on the proof of the asymptotic stability of the Delta/Par4-like robot system controlled by the ANPD controller (21). Before proceeding, two lemmas are introduced based on $[11,15]$.

Definition 1. A scalar continuous function $\alpha(x)$, defined for $x \in[0, a)$, is said to belong to the class $\mathscr{K}$ if it is strictly increasing and $\alpha(0)=0[16]$.

Lemma 2. Let the function $\alpha_{i}($.$) belongs to the class \mathscr{K}$ and (.) : $\mathscr{R} \longrightarrow \mathscr{R}$ is a continuous function. If $(x) \geq \alpha(|x|) \forall x \in$ $\mathscr{R}$, then

$$
\int_{0}^{x} f(\tau) d \tau>0, \forall x \neq 0 \in \mathscr{R}
$$

and

$$
\int_{0}^{x} f(\tau) d \tau \longrightarrow \infty \quad \text { as }|x| \longrightarrow \infty
$$

The concept of Lemma 2 can be clarified using Figure 5.

Proof. Since $\alpha_{i}$ (.) belongs to the class $\mathscr{K}$, then $\alpha(|x|)>0$, which means that $\alpha(|x|)$ is a real-valued function. Additionally, since $f(x) \geq \alpha(|x|)$, then the area under the curve defined by $\int_{0}^{x} f(\tau) d \tau$ yields strictly positive real value, which proves Lemma 2.

Lemma 3. The continuous matrix diagonal $K_{p}$ can be considered as $\mathscr{R}^{3} \longrightarrow \mathscr{R}^{3 \times 3}$

$$
\boldsymbol{K}_{p}(\boldsymbol{e})=\left[\begin{array}{ccc}
K_{p}\left(e_{1}\right) & 0 & 0 \\
0 & K_{p}\left(e_{2}\right) & 0 \\
0 & 0 & K_{p}\left(e_{3}\right)
\end{array}\right]
$$

where $e_{i}=e_{x}, e_{y}, e_{z}$ for $i=1,2,3$, respectively.
Suppose that there exist class $\mathscr{K}$ function $\alpha_{i}($.$) such that$

$$
f\left(e_{i}\right) \geq \alpha_{i}\left(\left|e_{i}\right|\right) \quad x \in \mathscr{R} i=1,2,3
$$

To guarantee a positive value of the function $f\left(e_{i}\right)$, it can be described as

$$
f\left(e_{i}\right)=e_{i} K_{p}\left(e_{i}\right)= \begin{cases}k_{p} e_{i}\left|e_{i}\right|^{\alpha_{1}-1}, & \left|e_{i}\right|>\delta_{1} \\ k_{p} e_{i} \delta_{1}^{\alpha_{1}-1}, & \left|e_{i}\right| \leq \delta_{1}\end{cases}
$$

In order to satisfy (31), then $\alpha_{i}\left(\left|e_{i}\right|\right)$ is expressed as

$$
\alpha_{i}(|x|)=\varepsilon_{i} e_{i} K_{p}\left(e_{i}\right) \quad 0<\varepsilon_{i}<1
$$

or

$$
\alpha_{i}\left(\left|e_{i}\right|\right)= \begin{cases}\varepsilon_{i} k_{p} e_{i}\left|e_{i}\right|^{\alpha_{i}-1}, & \left|e_{i}\right|>\delta_{1} \\ \varepsilon_{i} e_{i} \delta_{i}^{\alpha_{i}-1}, & \left|e_{i}\right| \leq \delta_{1}\end{cases}
$$

Referring to Lemma 2 and since $f\left(e_{i}\right)>0$, we have

$$
\int_{0}^{e_{i}} f\left(\eta_{i}\right) d \eta_{i}>0, \quad \forall e_{i} \neq 0 \in \mathscr{R}
$$

Consequently,

$$
\int_{0}^{e_{i}} \eta_{i} K_{p}\left(\eta_{i}\right) d \eta_{i}>0, \quad \forall e_{i} \neq 0 \in \mathscr{R}
$$

and

$$
\int_{0}^{e_{i}} \eta_{i} K_{p}\left(\eta_{i}\right) d \eta_{i} \longrightarrow \infty \quad\left|e_{i}\right| \longrightarrow \infty
$$

If we set $\boldsymbol{\eta}^{\boldsymbol{T}}=\left[\begin{array}{lll}\eta_{1} & \eta_{2} & \eta_{2}\end{array}\right]^{T}$, then the function

$$
\begin{aligned}
\int_{0}^{e} \boldsymbol{\eta}^{\boldsymbol{T}} \boldsymbol{K}_{p}(\boldsymbol{\eta}) d \boldsymbol{\eta}= & \int_{0}^{e_{1}} \eta_{1} K_{p 1}\left(\eta_{1}\right) d \eta_{1} \\
& +\int_{0}^{e_{2}} \eta_{2} K_{p}\left(\eta_{2}\right) d \eta_{2} \\
& +\int_{0}^{e_{3}} \eta_{3} K_{p}\left(\eta_{3}\right) d \eta_{3}
\end{aligned}
$$

is a positive definite function. Figure 6 illustrates graphically the concept of Lemma 3 proof.

Theorem 4. If $K_{p}($.$) and K_{d}($.$) are nonlinear gains defined$ by (26) and (27), respectively, then the closed-loop Delta/Par4like robot system based on ANPD control law, given by (21), is asymptotically stable.

Proof. Let us candidate a Lyapunov function of the following form:

$$
V(\boldsymbol{e}, \dot{\boldsymbol{e}})=\frac{1}{2} \dot{\boldsymbol{e}} \boldsymbol{M} \dot{\boldsymbol{e}}^{T}+\int_{0}^{e} \boldsymbol{\eta}^{T} \boldsymbol{K}_{p}(\boldsymbol{\eta}) d \boldsymbol{\eta}
$$

Due to the positive definiteness of matrix $\boldsymbol{M}$, the term $(1 / 2) \dot{\boldsymbol{e}} \boldsymbol{M} \dot{\boldsymbol{e}}^{T}$ is a positive definite function. The second term 


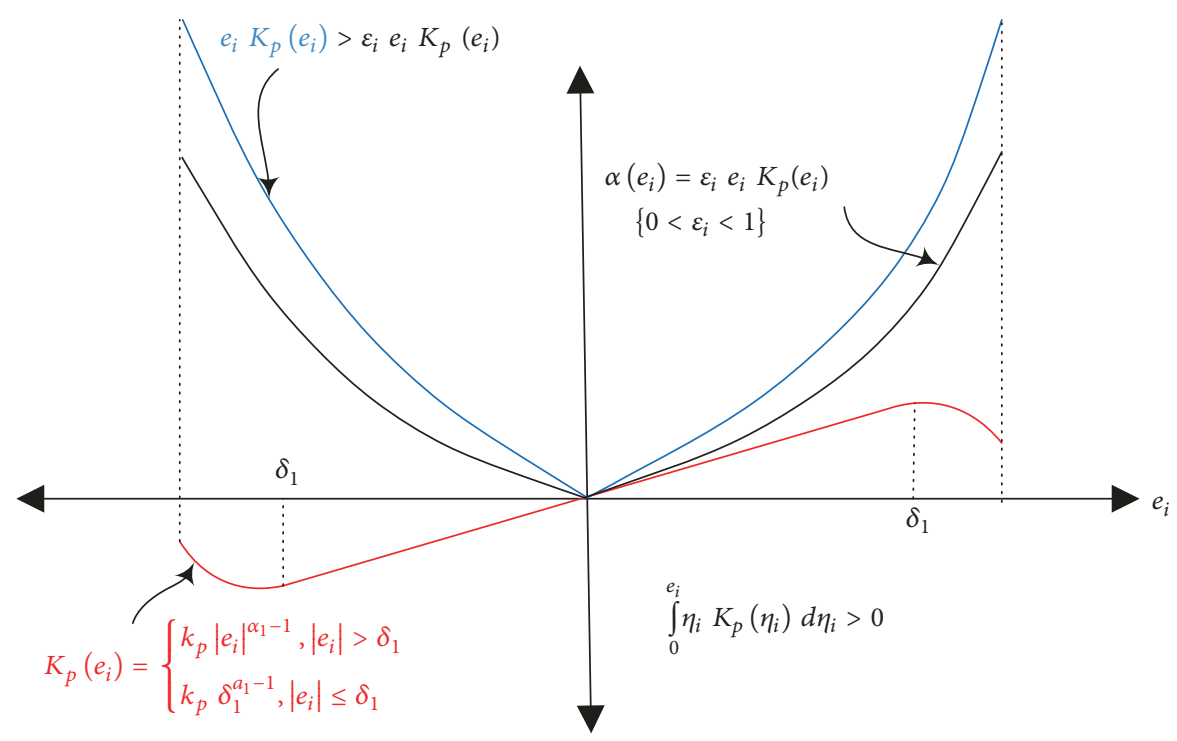

FIGURE 6: Graphical description of Lemma 3.

of Lyapunov function $\int_{0}^{e} \boldsymbol{\eta}^{\boldsymbol{T}} \boldsymbol{K}_{p}(\boldsymbol{\eta}) d \boldsymbol{\eta}$ can be explained as the potential energy, which is driven by error part of the controller and it is given by (38) and it has been shown that it is unbounded positive definite. Therefore, $V(e, \dot{e})$ is a positive definite function.

The time derivative of the Lyapunov function is

$$
\dot{V}(e, \dot{e})=\dot{\boldsymbol{e}}^{T} \boldsymbol{M} \ddot{\boldsymbol{e}}+\frac{1}{2} \dot{\boldsymbol{e}}^{T} \dot{\boldsymbol{M}} \dot{\boldsymbol{e}}+\boldsymbol{e}^{T} \boldsymbol{K}_{p}(\boldsymbol{e}) \dot{\boldsymbol{e}}
$$

Using the control law defined by (21) and the dynamic model given by (15), the closed-loop system equation becomes

$$
\boldsymbol{M} \ddot{\boldsymbol{X}}+C \dot{\boldsymbol{X}}=\boldsymbol{M} \ddot{\boldsymbol{X}}^{d}+C \dot{\boldsymbol{X}}^{d}+K_{p}(\boldsymbol{e}) \boldsymbol{e}+K_{d}(\dot{\boldsymbol{e}}) \dot{\boldsymbol{e}}
$$

or

$$
\begin{aligned}
& \boldsymbol{M}\left(\ddot{\boldsymbol{X}}^{d}-\ddot{\boldsymbol{X}}\right)+C\left(\dot{\boldsymbol{X}}^{d}-\dot{\boldsymbol{X}}\right)+\boldsymbol{K}_{\boldsymbol{p}}(\boldsymbol{e}) \boldsymbol{e}+\boldsymbol{K}_{d}(\dot{\boldsymbol{e}}) \dot{\boldsymbol{e}} \\
& \quad=[0]
\end{aligned}
$$

Equation (42) can be rewritten as

$$
M \ddot{e}+C \dot{e}+K_{p}(e) e+K_{d}(\dot{e}) \dot{e}=[0]
$$

where $\boldsymbol{e}$ is the position error, $\dot{\boldsymbol{e}}=\dot{\boldsymbol{X}}^{d}-\dot{\boldsymbol{X}}$, and $\ddot{\boldsymbol{e}}=\ddot{\boldsymbol{X}}^{d}-\ddot{\boldsymbol{X}}$.

Multiplying both sides of (43) by $\dot{\boldsymbol{e}}^{T}$ from the left and solving for $\dot{\boldsymbol{e}}^{T} \boldsymbol{M} \ddot{\boldsymbol{e}}$ to have

$$
\dot{\boldsymbol{e}}^{T} \boldsymbol{M} \ddot{\boldsymbol{e}}=-\dot{\boldsymbol{e}}^{T} \boldsymbol{C} \dot{\boldsymbol{e}}-\dot{\boldsymbol{e}}^{T} \boldsymbol{K}_{\boldsymbol{p}}(\boldsymbol{e}) \boldsymbol{e}-\dot{\boldsymbol{e}}^{T} \boldsymbol{K}_{\boldsymbol{d}}(\dot{\boldsymbol{e}}) \dot{\boldsymbol{e}}
$$

Substituting the result from (44) into (40), we have

$$
\begin{aligned}
\dot{V}(e, \dot{e})= & -\dot{\boldsymbol{e}}^{T} \boldsymbol{C} \dot{\boldsymbol{e}}-\dot{\boldsymbol{e}}^{T} \boldsymbol{K}_{p}(\boldsymbol{e}) \boldsymbol{e}-\dot{\boldsymbol{e}}^{T} \boldsymbol{K}_{\boldsymbol{d}}(\dot{\boldsymbol{e}}) \dot{\boldsymbol{e}} \\
& +\frac{1}{2} \dot{\boldsymbol{e}}^{T} \dot{\boldsymbol{M}} \dot{\boldsymbol{e}}+\boldsymbol{e}^{T} \boldsymbol{K}_{p}(\boldsymbol{e}) \dot{\boldsymbol{e}}
\end{aligned}
$$

Since $\boldsymbol{K}_{p}(\boldsymbol{e})$ is a diagonal matrix, the terms $-\dot{\boldsymbol{e}}^{T} \boldsymbol{K}_{p}(\boldsymbol{e}) \boldsymbol{e}$ and $\boldsymbol{e}^{T} \boldsymbol{K}_{p}(\boldsymbol{e}) \dot{\boldsymbol{e}}$ are cancelled out and (45) reduces

$$
\dot{V}=-\dot{\boldsymbol{e}}^{T} \boldsymbol{K}_{d}(\dot{\boldsymbol{e}}) \dot{\boldsymbol{e}}+\frac{1}{2} \dot{\boldsymbol{e}}^{T}(\dot{\boldsymbol{M}}-2 \boldsymbol{C}) \dot{\boldsymbol{e}}
$$

Since the property $\dot{\boldsymbol{e}}^{T}(\dot{\boldsymbol{M}}-2 \boldsymbol{C}) \dot{\boldsymbol{e}}=0$ is satisfied in the considered robot, (46) becomes

$$
\dot{V}=-\dot{\boldsymbol{e}}^{T} K_{d}(\dot{\boldsymbol{e}}) \dot{\boldsymbol{e}}
$$

Since $K_{d}(\dot{\boldsymbol{e}})$ is a symmetric positive definite matrix, then the function $\dot{V}$ is seminegative definite and hence Delta/Par4-like robot is stable.

However, since $V(t)>0$ and $\dot{V}(t) \leq 0$, the asymptotic stability of the origin is not guaranteed and the trajectory may not reach to equilibrium at the origin, but it converges to specified limit instead.

Based on Lyapunov function $V(t)$ described by (39), one may conclude that both of error and error derivative is bounded. Since the matrix $\boldsymbol{M}$ is symmetric positive definite, therefore its inverse $M^{-1}$ exists and bounded. Then, the closed-loop error dynamic systems can be written as

$$
\ddot{\boldsymbol{e}}=-\boldsymbol{M}^{-1}\left(\boldsymbol{C} \dot{\boldsymbol{e}}+\boldsymbol{K}_{p}(\boldsymbol{e}) \boldsymbol{e}+\boldsymbol{K}_{d}(\dot{\boldsymbol{e}}) \dot{\boldsymbol{e}}\right)
$$

According to Barbalat lemma [17], the second derivative of error $\ddot{e}$ is also bounded. Therefore, one can decisively conclude $\dot{e} \longrightarrow \infty$ as $\mathrm{t} \longrightarrow 0$, which implies that $e \longrightarrow \infty$ as $t \longrightarrow 0$.

The block diagram of Delta/Par4-like Robot control scheme based on augmented PD controller is shown in Figure 7. As indicated earlier, this controller is composed of two parts: the first part is the PD-based control law and the second part is the feedback signal. Moreover, the PDbased control can be either classical PD controller (APD) or 


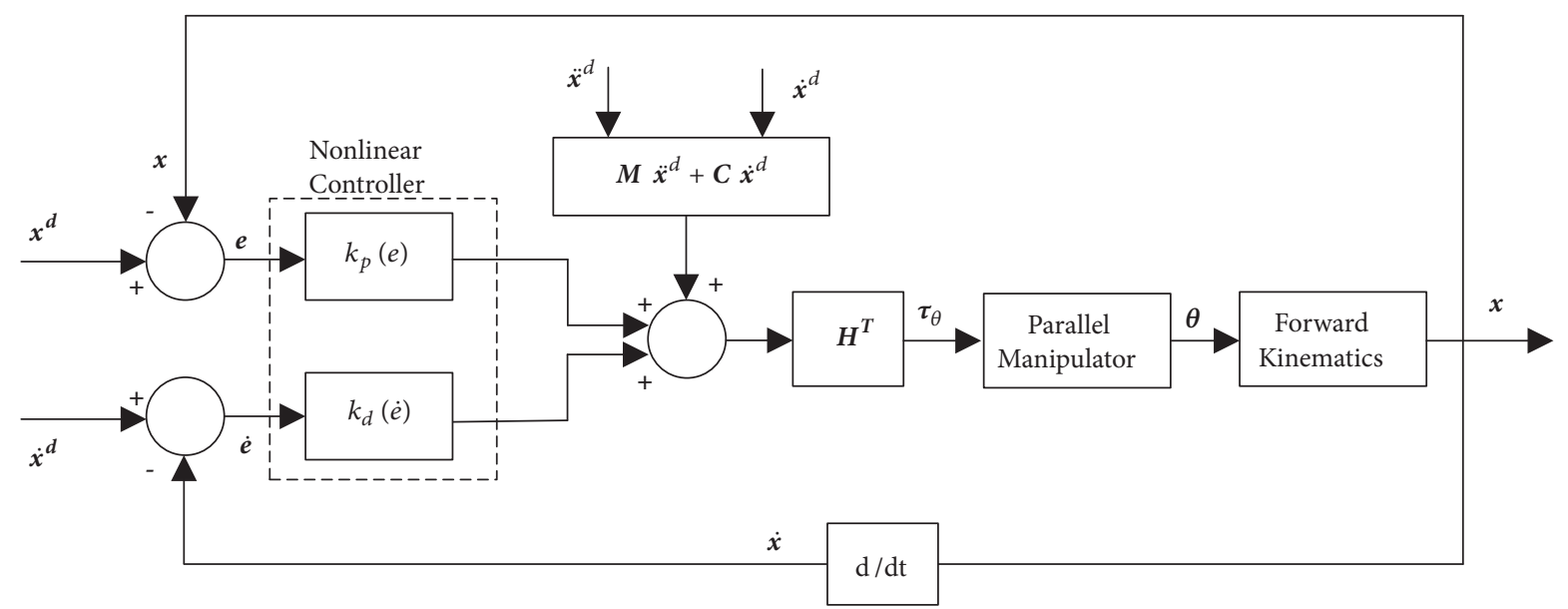

FIGURE 7: Block diagram of ANPD controller.

TABLE 1: Numerical values of system parameters [10].

\begin{tabular}{lcc}
\hline The Parameters & Symbols & Values \\
\hline The radius of robot base. & $R_{b}$ & $0.35(\mathrm{~m})$ \\
Traveling plate radius. & $R_{t p}$ & $0.1(\mathrm{~m})$ \\
Arm length. & $l_{A}$ & $0.35(\mathrm{~m})$ \\
Forearm length. & $l_{B}$ & $0.8(\mathrm{~m})$ \\
Traveling plate mass. & $m_{t p}$ & $0.6(\mathrm{~kg})$ \\
The inertia of actuating motor. & $I_{a c t}$ & $0.003\left(\mathrm{~kg} \cdot \mathrm{m}^{2}\right)$ \\
Arm inertia. & $I_{\text {arm }}$ & $0.071\left(\mathrm{~kg} \cdot \mathrm{m}^{2}\right)$ \\
Forearm Mass. & $m_{f}$ & $0.3(\mathrm{~kg})$ \\
The maximum allowable torque. & $\tau_{q_{\max }}$ & $90(\mathrm{~N} . \mathrm{m})$ \\
\hline
\end{tabular}

nonlinear PD controller (ANPD). The actuating control input for augmented PD controller can be written as

$$
\boldsymbol{\tau}_{q}=\boldsymbol{H}^{T}\left(\boldsymbol{M} \ddot{\boldsymbol{X}}^{d}+\boldsymbol{C} \dot{\boldsymbol{X}}^{d}+K_{p}(\boldsymbol{e}) \boldsymbol{e}+K_{d}(\dot{\boldsymbol{e}}) \dot{\boldsymbol{e}}\right)
$$

where $\boldsymbol{H}^{T}$ is pseudoinverse of $J_{m}^{T}$.

In case of nonaugmented PD controller, the terms $\boldsymbol{M} \ddot{\boldsymbol{X}}^{d}$ and $\boldsymbol{C} \dot{\boldsymbol{X}}^{d}$ are eliminated from (49), which becomes

$$
\boldsymbol{\tau}_{q}=\boldsymbol{H}^{T}\left(K_{p}(\boldsymbol{e}) \boldsymbol{e}+K_{d}(\dot{\boldsymbol{e}}) \dot{\boldsymbol{e}}\right)
$$

In addition to performance comparison between the augmented PD structures (APD and ANPD), a comparison is made between augmented nonlinear PD (ANPD) controller and nonaugmented nonlinear PD (NANPD) controller.

\section{Simulation Results}

Delta/Par4-like robot is redundant parallel manipulator consisting of four motors and 3-DOF, and its actuators are the RTMB140-100 ETEL, which have a maximum torque $127 \mathrm{~N}$. $\mathrm{m}$ and maximum speed $550 \mathrm{RPM}$ and workspace (cylinder of $300 \mathrm{~mm}$ radius and $100 \mathrm{~mm}$ height) [8]. The numeric values of system parameters are listed in Table 1.
TABLE 2: Settings of controller parameters.

\begin{tabular}{lccc}
\hline $\begin{array}{l}\text { Parameters of } \\
\text { ANPD }\end{array}$ & Value & $\begin{array}{c}\text { Parameters of } \\
\text { APD }\end{array}$ & Value \\
\hline$k_{p}$ & {$[1,1,1] \times 10^{5}$} & $k_{p}$ & $2 \times 10^{4}$ \\
\hline$k_{d}$ & {$[5,5,5] \times 10^{3}$} & $k_{d}$ & 150 \\
\hline$\alpha_{1}$ & {$[1,1,1] \times 10^{-1}$} & & \\
\hline$\alpha_{2}$ & {$[5,5,5] \times 10^{-1}$} & & \\
\hline$\delta_{1}$ & {$[9,0,3] \times 10^{-4}$} & & \\
\hline$\delta_{2}$ & {$[416,0,324] \times 10^{-4}$} & & \\
\hline
\end{tabular}

In this section, the dynamic model of Delta/par4-like robot and proposed controllers are implemented in Matlab/Simulink (R2017b). The setting of parameters for both ANPD and APD structures is based on the try-and-error procedure and listed in Table 2. by

The desired trajectory in Cartesian space can be described

$$
\begin{aligned}
x(t) & =0.15 \cdot \sin \left(6 \pi t+\frac{3 . \pi}{2}\right), \\
y(t) & =0, \\
z & =-0.53
\end{aligned}
$$

Figure 8 depicts the traces of above desired trajectory.

In performance evaluation of accuracy in most robotic applications, the RMSE is used instead of Integral absolute value of error (IAE) or Integral of the square value of error (ISE). However, the RMSE criteria is strongly related to ISE one, where RMSE $=\sqrt{\left(\int e^{2} d t\right)}$. In the present work, a discrete version of RMSE is adopted to evaluate the accuracy performance, which can be defined by

$$
\begin{aligned}
\operatorname{RMSE} & =\sqrt{\left.\frac{1}{N} \sum_{j=1}^{N}\left(e_{x}^{2}(j)+e_{y}^{2}(j)\right)+e_{z}^{2}(j)\right)} \\
& =\sqrt{\frac{1}{N} \sum_{j=1}^{N}\left(x^{d}(j)-x(j)\right)^{2}+\left(y^{d}(j)-y(j)\right)^{2}+\left(z^{d}(j)-z(j)\right)^{2}}
\end{aligned}
$$




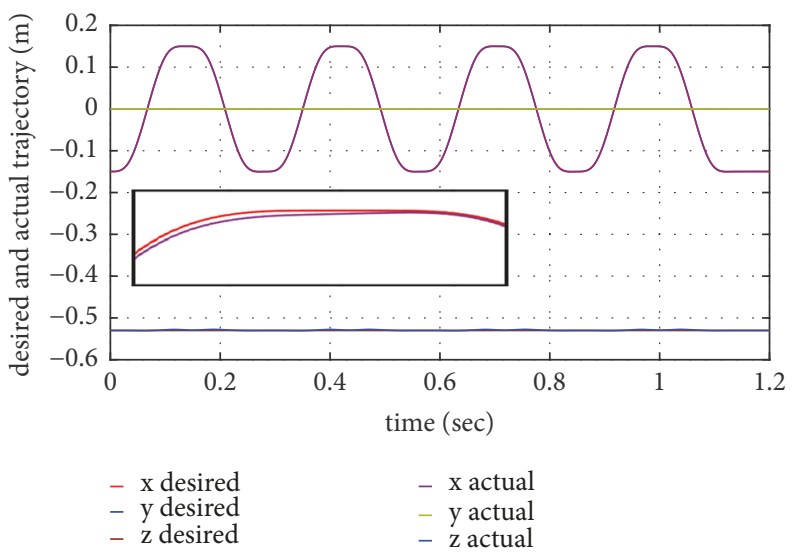

FIGURE 8: The traces of the desired trajectory.

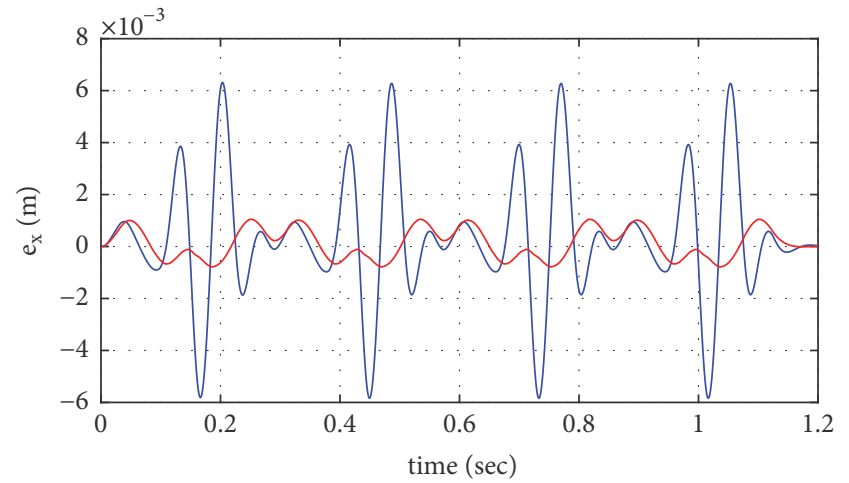

- APD

- ANPD

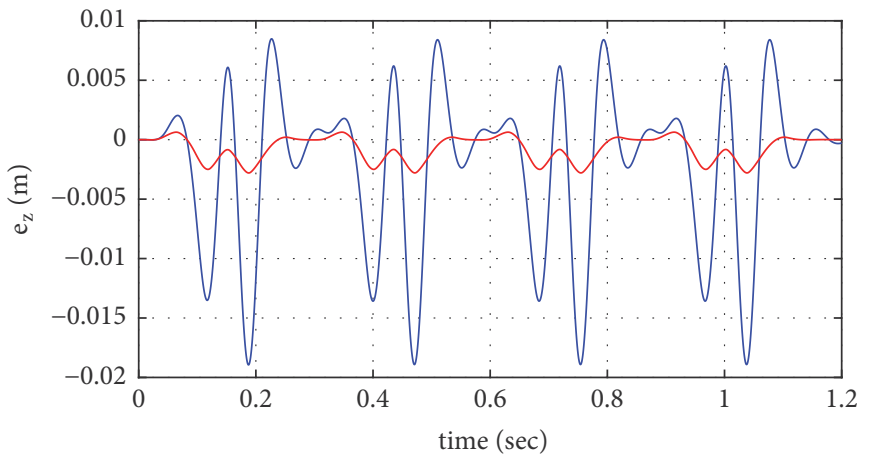

- APD

- ANPD

(a)

(b)

FIGURE 9: Cartesian error dynamics in $x$ and $z$ based on APD and ANPD.

TABLE 3: The RMSE given by the APD and ANPD controllers.

\begin{tabular}{lcc}
\hline APD & ANPD & Improvement \\
\hline 0.0068 & 0.0013 & $78.27 \%$ \\
\hline
\end{tabular}

where $N$ is the number of samples, $j$ is the sampling instant, and $e_{x}, e_{y}$, and $e_{z}$ represent the tracking errors along $x, y$, and $z$.

The first part of the simulation establishes a comparison study in performance between augmented PD control structures (APD and ANPD). Figure 9 shows the error behaviors on $\mathrm{x}, \mathrm{y}$ and $\mathrm{z}$-axes for APD and ANPD controllers. It is clear from the figure that the ANPD controller can provide better tracking performance than the APD controller. Also, it is worthy to notice that the errors in $y$-axis given by both controllers are in order of $10^{-17}$, which can be regarded as a negligible error. However, this is a reasonable result since there is no desired trajectory on this axis $y(t)=0$. As such this axis is discarded from the comparison in the next part of simulation.

Table 3 reports the performances of APD and ANPD controllers and gives the percentage of improvement gained with ANPD controller. The better controller is the one which gives less RMSE value. From Table 3, the RMSE value of error given by ANPD is equal to 0.0013 , while that resulting from APD is equal to 0.0068 with an improvement of $78.27 \%$. Therefore, one can conclude that ANPD gives better tracking accuracy with an improvement of $78.27 \%$.

The responses of torques generated by the four robot actuators are shown in Figure 10. These control efforts, represented by torque signals have to respond to the requirements of controllers such as to compensate and pay for accuracy improvement.

The second part of simulation results focuses on the evaluation of performance based on augmented and nonaugmented nonlinear PD controllers (ANPD and NANPD). Figure 11 shows the behaviors of error on $x$ and $z$-axes for ANPD and NANPD controllers. Table 3 lists the values of RMSE for ANPD and NANPD. It is evident from the table that ANPD results in a value of RMSE equals to 0.0013 , while a value of 0.0052 is resulting from NANPD controller. This indicates that ANPD controller achieves 75\% improvement and it outperforms NANPD controller in terms of tracking performance. 


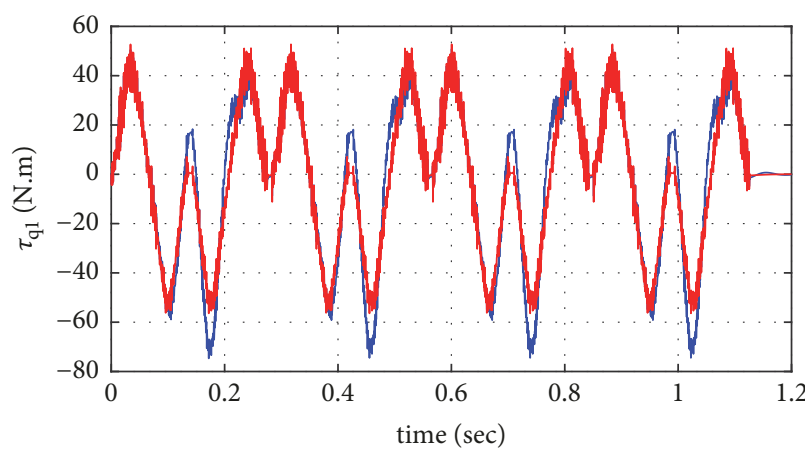

- APD
- ANPD

(a)

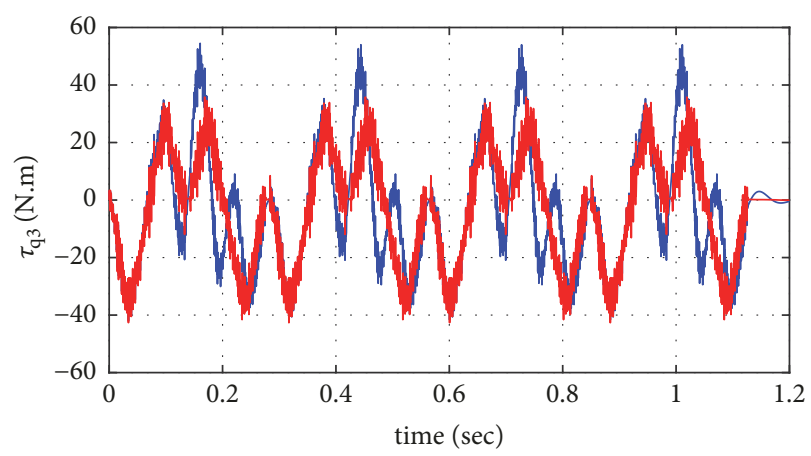

- APD
- ANPD

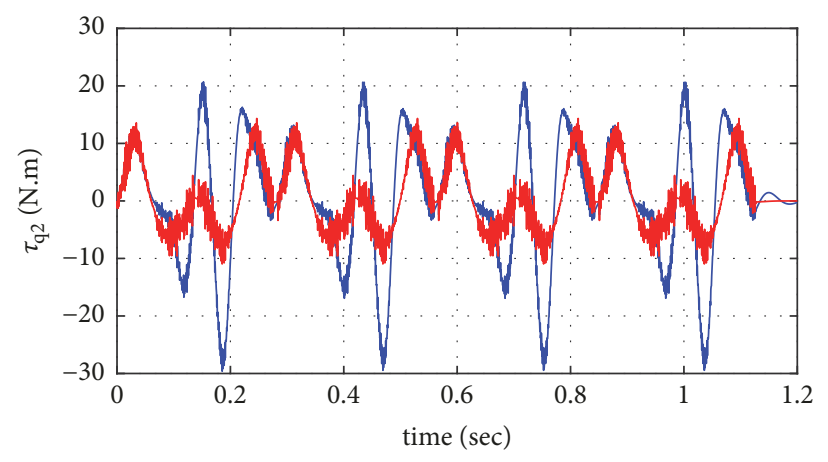

- APD

- ANPD

(b)

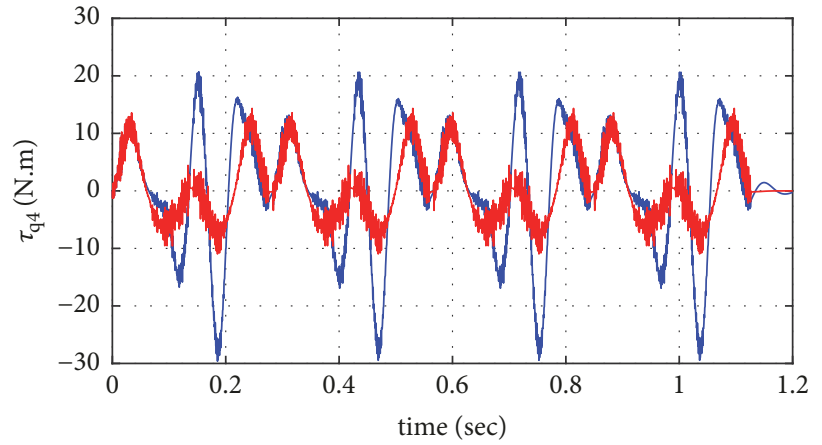

- APD

- ANPD

(c)

(d)

FIgURE 10: Torque responses for APD and ANPD controllers.

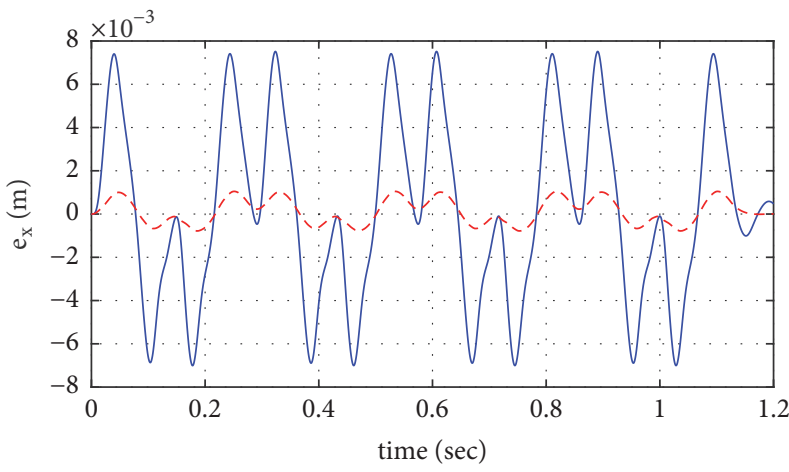

- - NPD

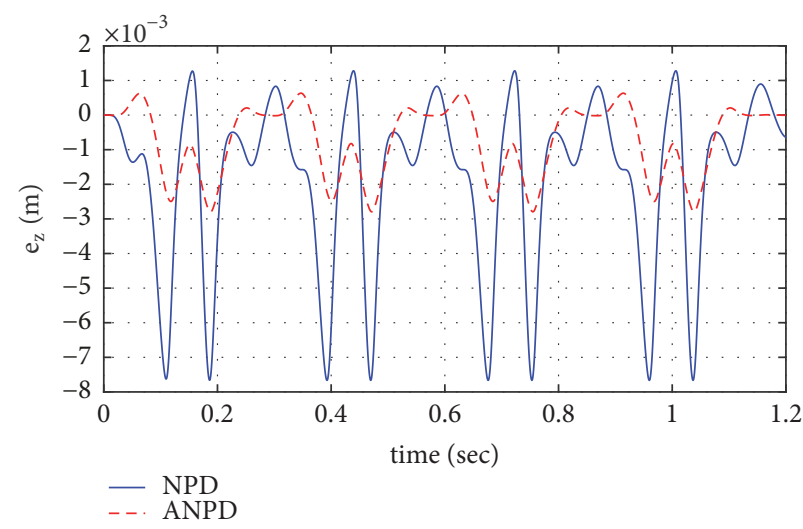

(b)

FIGURE 11: Cartesian error dynamic in $x$ - and $z$-axes for NANPD and ANPD.

The torque responses generated from the four actuating motors of the parallel robot is depicted in Figure 12. For a fair comparison, the same set of parameters given to ANPD, which are listed in Table 2, are assigned to the corresponding parameters of NANPD controller.

\section{Conclusion}

This paper presented two comparison studies. One study is based on the comparison between augmented PD control structures (APD and NAPD controller). The other comparison study is established between augmented nonlinear 


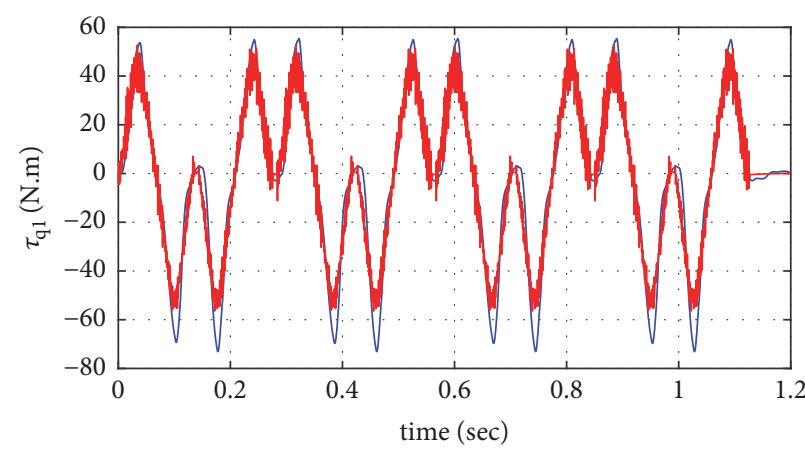

- NPD

- ANPD

(a)

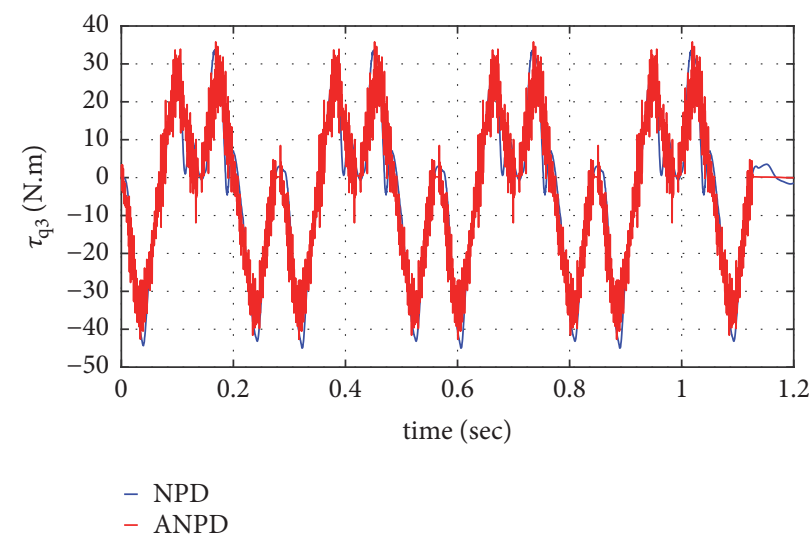

(c)

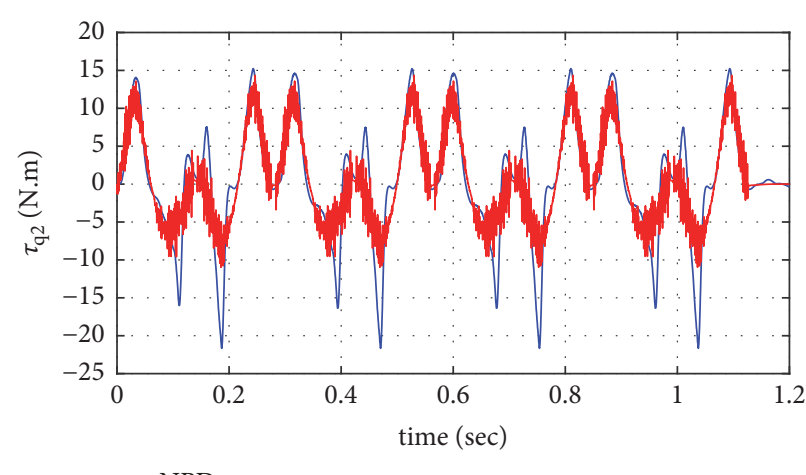

- NPD

- ANPD

(b)

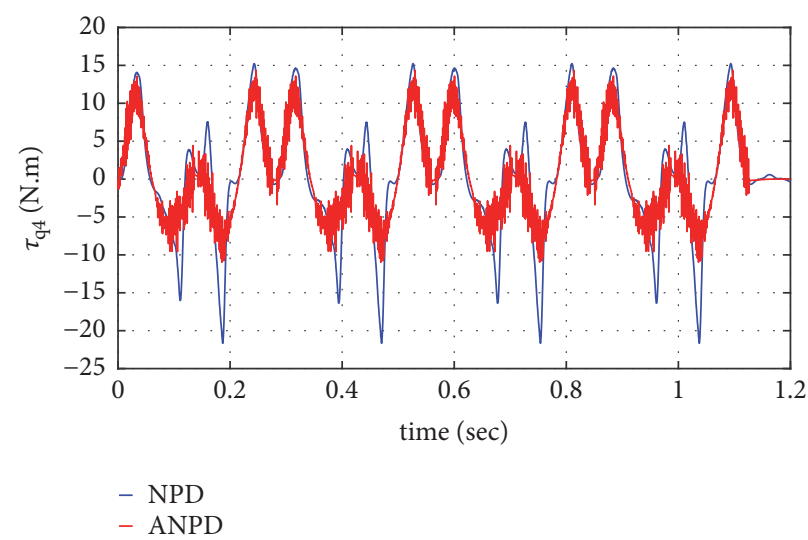

(d)

FIgURE 12: Torque responses for NANPD and ANPD controllers.

TABLE 4: The RMSE given by the NANPD and ANPD controllers.

\begin{tabular}{lcc}
\hline NANPD & ANPD & Improvement \\
\hline 0.0052 & 0.0013 & $75 \%$ \\
\hline
\end{tabular}

PD (ANPD) controller and nonaugmented nonlinear PD (NANPD) controller. The assessment of controllers for comparison is made in terms of tracking performance and accuracy for 3-DOF Delta/Par4-like parallel robot. The measure of improvement is calculated in terms of error variance.

The simulated results showed that ANPD controller gives 78.26\% improvement in tracking accuracy as compared to that given by APD as indicated by Table 3. Also, the ANPD controller gives 75\% improvement in performance as compared to that given by NANPD as reported by Table 4 . Therefore, one can conclude that ANPD outperforms both APD and NANPD controllers.

\section{Data Availability}

No data were used to support this study.

\section{Conflicts of Interest}

The authors declare that they have no conflicts of interest.

\section{References}

[1] R. Jee-Hwan, Parallel Manipulators-New Development, I-Tech Education and Publishing, Vienna, Austria, 2008.

[2] J. Q. Han, "From PID to active disturbance rejection control," IEEE Transactions on Industrial Electronics, vol. 56, no. 3, pp. 900-906, 2009.

[3] R. Anoop and K. Achu, "Control technique for parallel manipulator using PID," International Journal of Engineering Research \& Technology, vol. 5, no. 7, pp. 56-59, 2016.

[4] Y.-X. Zhang, S. Cong, W.-W. Shang, Z.-X. Li, and S.-L. Jiang, "Modeling, identification and control of a redundant planar 2-DOF parallel manipulator," International Journal of Control, Automation, and Systems, vol. 5, no. 5, pp. 559-569, 2007.

[5] H. Saied, A. Chemori, M. El Rafei, C. Francis, and F. Pierrot, "From non-model-based to model-based control of pkms: a comparative study," in Proceedings of the 1st International Congress for the Advancement of Mechanism, Machine, Robotics and Mechatronics Sciences, pp. 50-64, Beirut, Lebanon, 2017.

[6] W. W. Shang, S. Cong, Z. X. Li, and S. L. Jiang, "Augmented nonlinear PD controller for a redundantly actuated parallel manipulator," Advanced Robotics, vol. 2, no. 23, pp. 1725-1742, 2009.

[7] S. Wei-Wei and C. Shuang, "Nonlinear computed torque control for a high-speed planar parallel manipulator," Mechatronics, vol. 19, no. 6, pp. 987-992, 2009. 
[8] Y. X. Su, B. Y. Duan, and C. H. Zheng, "Nonlinear PID control of a six-DOF parallel manipulator," IEE Proceedings, Control Theory and Applications, vol. 151, no. 1, pp. 95-102, 2004.

[9] F. Abdelhedi, Y. Bouteraa, A. Chemori, and N. Derbel, "Nonlinear PID and feed-forward control of robot manipulator," in Proceedings of the 15th International Conference on Sciences and Techniques of Automatic Control and Computer Engineering, STA 2014, pp. 349-354, IEEE, Hammamet, Tunisia, 2014.

[10] D. Corbel, M. Gouttefarde, O. Company, and F. Pierrot, "Toward 100G with PKM: is actuation redundancy a good solution for pick and place?" in Proceedings of the 2010 IEEE International Conference on Robotics and Automation, ICRA 2010, pp. 46754682, IEEE, Anchorage, Alaska, USA, May 2010.

[11] G. Sartori Natal, A. Chemori, and F. Pierrot, "Dual-space adaptive control of redundantly actuated parallel manipulator for extremely fast operations with load changes," in Proceedings of the 2012 IEEE International Conference on Robotics and Automation, ICRA 2012, pp. 253-258, IEEE, Saint Paul, Minn, USA, May 2012.

[12] F. Pierrot, C. Baradat, V. Nabat, O. Company, S. Krut, and M. Gouttefarde, "Above $40 \mathrm{~g}$ acceleration for pick-and-place with a new 2-DOF PKM," in Proceedings of the 2009 IEEE International Conference on Robotics and Automation, ICRA '09, pp. 17941800, IEEE, Kobe, Japan, May 2009.

[13] V. Nabat, Robots parallèles à nacelle articulée, du concept à la solution industrielle pour le pick-and place [Ph.D. thesis], Montpellier 2 University, Montpellier, France, 2007.

[14] J. Q. Han, "Nonlinear PID controller," Acta Mathematica Sinica, vol. 20, no. 4, pp. 487-490, 1994.

[15] R. Kelly and R. Carelli, "A class of nonlinear PD-type controllers for robot manipulators," Journal of Robotic Systems, vol. 13, no. 19, pp. 793-802, 1996.

[16] K. Hassan, Nonlinear Control, Pearson, London, UK, Global Edition edition, 2015.

[17] R. Kelly, V. Santibez, and A. Lora, Control of Robot Manipulators in Joint Space, Springer-Verlag, London, UK, 2005. 


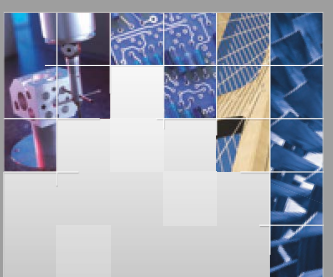

\section{Enfincering}
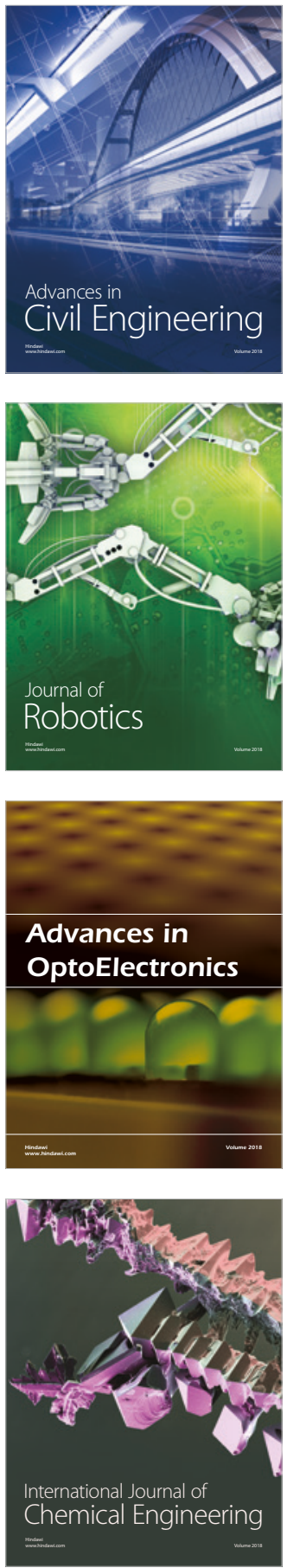

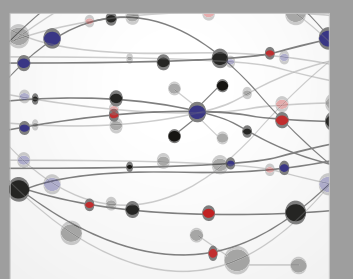

\section{Rotating \\ Machinery}

The Scientific World Journal

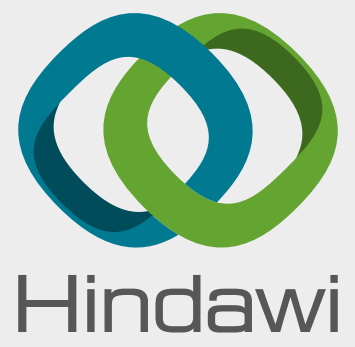

Submit your manuscripts at

www.hindawi.com
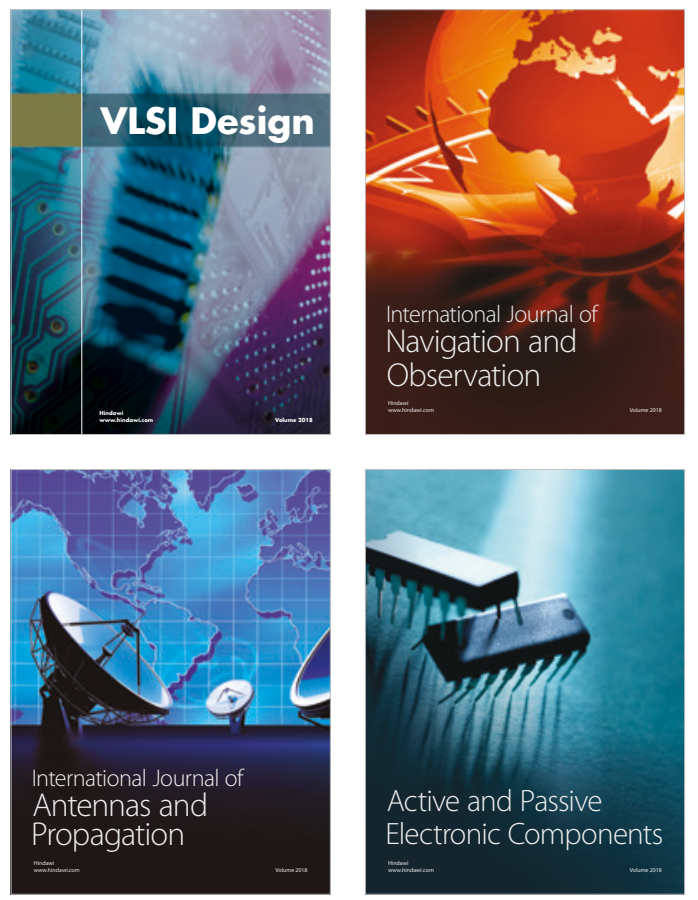
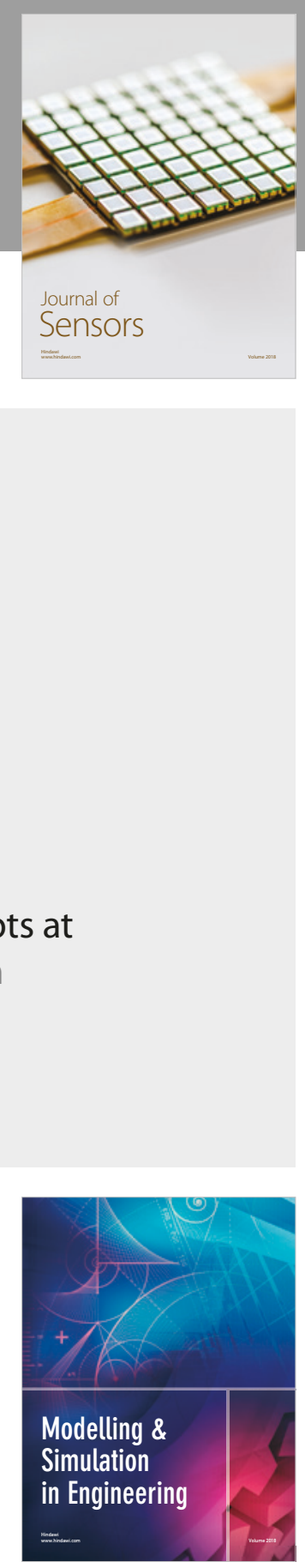

\section{Advances \\ Multimedia}
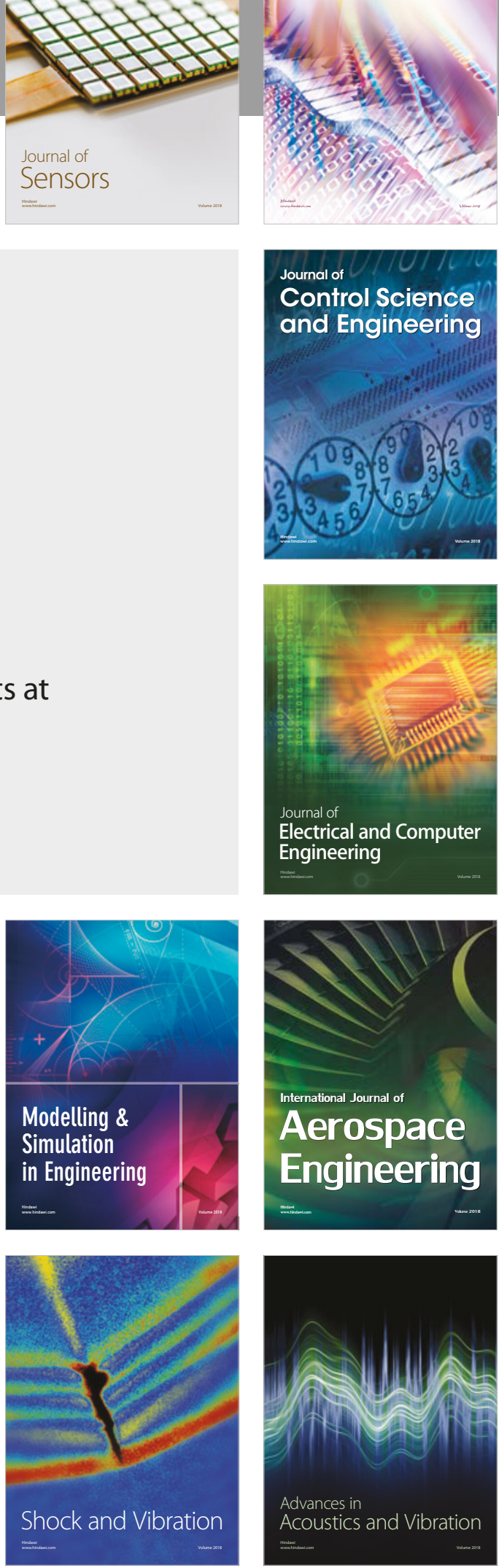